\title{
Decompression of malignant biliary obstruction by duodenoscopic intubation of bile duct
}

\author{
BERNARD H LAURENCE, PETER B COTTON
}

\section{Summary and conclusions}

Interest is increasing in non-operative methods of relieving malignant obstruction of the bile duct, and drainage tubes and prostheses may be placed in the bile duct via the percutaneous transhepatic route. Two cases are described, however, in which a duodenoscope was used and the approach was via the papilla of Vater. This method allows temporary or permanent drainage tubes to be placed through malignant strictures.

This endoscopic approach is less invasive and should be safer than that by the transhepatic route; furthermore, removing and replacing a blocked endoprosthesis should be easier. Further study is needed, as the procedure is technically more difficult and its role in managing biliary strictures has yet to be defined.

\section{Introduction}

Many patients present with biliary obstruction due to unresectable malignant tumours. Even palliative bypass procedures carry a high operative mortality in the presence of deep jaundice. ${ }^{12}$ This may be reduced by preoperative decompression with a percutaneous transhepatic catheter. ${ }^{3}$ The same route may be used to insert a permanent biliary prosthesis, obviating the need

Gastrointestinal Unit, Middlesex Hospital, London W1N 8AA

BERNARD H LAURENCE, FRACP, locum consultant physician (permanent appointment : consultant gastroenterologist, Sir Charles Gairdner Hospital, Perth, Western Australia)

PETER B COTTON, MD, FRCP, consultant physician for surgical bypass. ${ }^{45}$ Even this technique, however, carries appreciable hazards. ${ }^{3}$

Duodenoscopic cannulation of the papilla of Vater permits early radiological diagnosis and cytological confirmation of malignant obstructive jaundice and yields a low rate of complications. ${ }^{6}$ Endoscopic sphincterotomy may be used to improve drainage in patients with obstructing tumours of the papilla of Vater. ${ }^{7}$ We have used a duodenoscope to provide palliative drainage in patients with higher lesions by placing tubes through the malignant biliary strictures.

\section{Methods}

Diagnosis-Coagulation defects were corrected and antibiotics given parenterally before the procedure. Endoscopic retrograde cholangiography was performed in the conventional manner, but a large instrument (Olympus GFB2 gastroscope) was used because a wide biopsy channel was needed. The radiological diagnosis of malignant obstruction was confirmed by endoscopic brushing cytology from within the bile duct.

Transnasal biliary drainage-A standard Teflon endoscopy catheter, external diameter $1.2 \mathrm{mn}$, with the tapered tip removed and containing a Teflon-coated guide wire (038, William Cook) was passed deep into the bile duct up to the obstruction. By manipulating the catheter the guide wire was passed through the stricture into the intrahepatic ducts (fig 1). The guide wire was left in place and the standard catheter withdrawn over it and replaced with an angiographic catheter (7 French, William Cook) with a tapered tip and multiple terminal side holes. This catheter was then forced through the stricture over the guide wire until the tip lay at least $5 \mathrm{~cm}$ above the obstruction; the guide wire was removed and the endoscope carefully withdrawn over the catheter. The proximal end of the catheter was rerouted from the mouth to the nares, and bile drained by gravity. If bile flow stopped the position of the catheter was checked radiologically (fig 2 ) and the
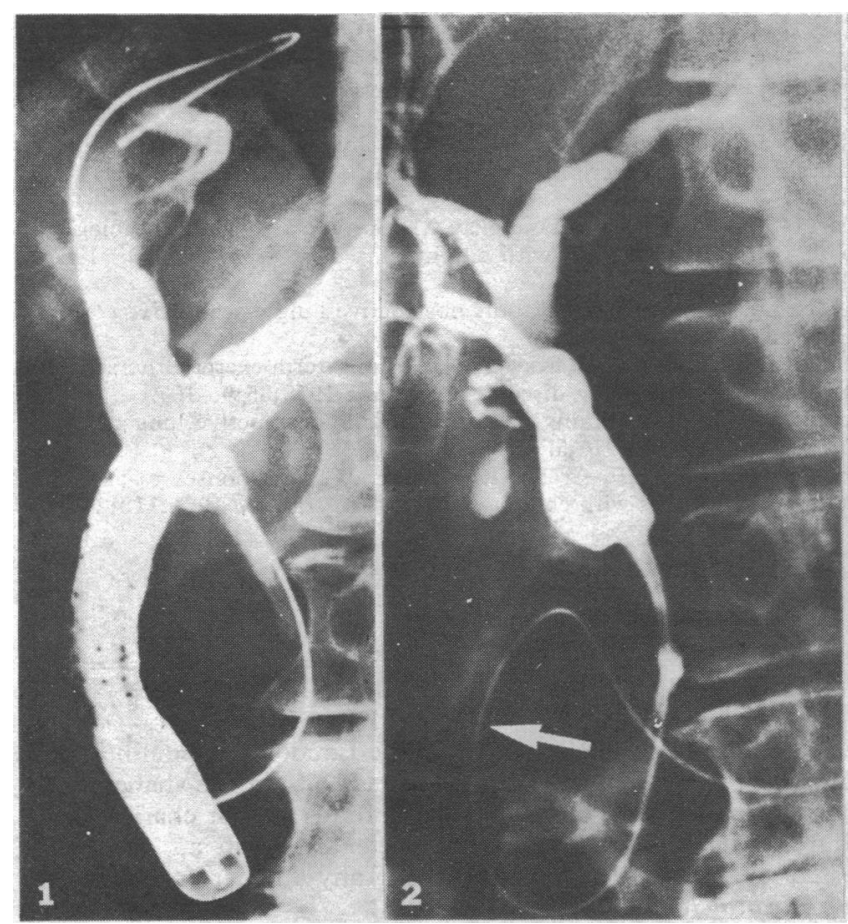
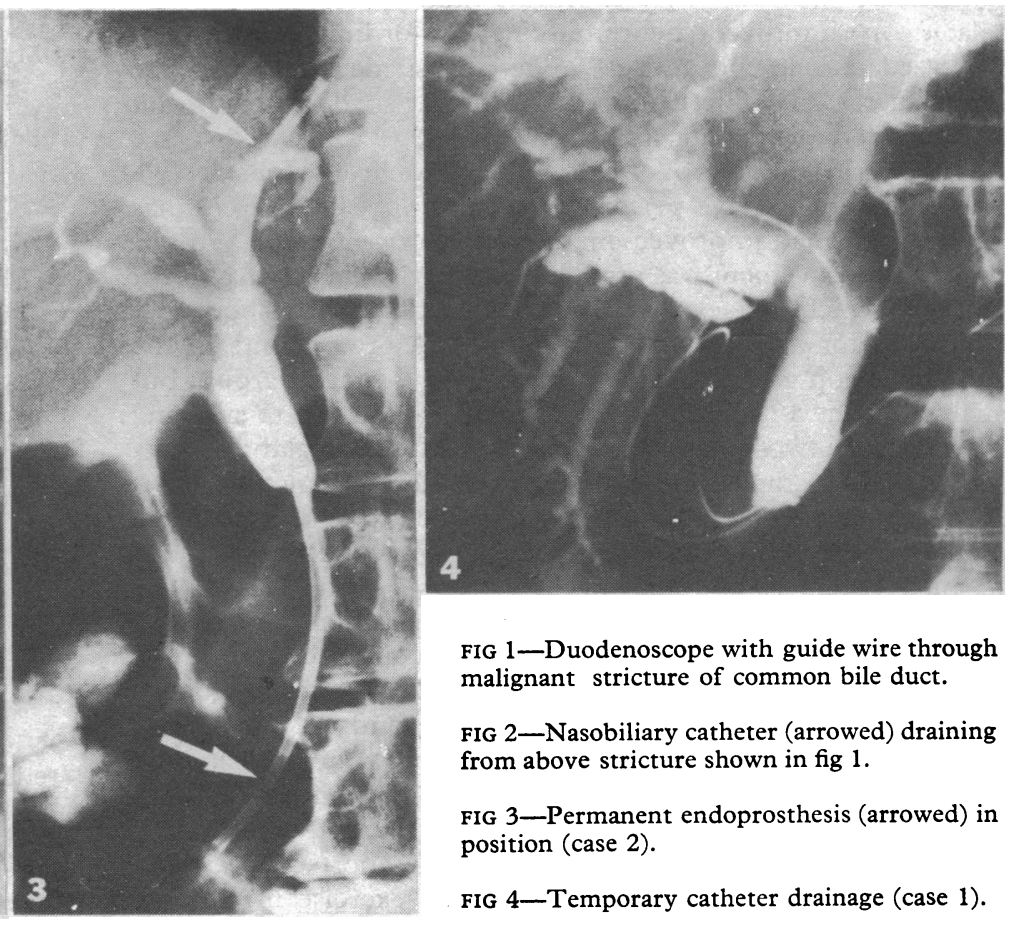

FIG 1-Duodenoscope with guide wire through malignant stricture of common bile duct.

FIG 2-Nasobiliary catheter (arrowed) draining from above stricture shown in fig 1 .

FIG 3-Permanent endoprosthesis (arrowed) in position (case 2).

FIG 4-Temporary catheter drainage (case 1). 
lumen cleared by passing a guide wire or by gentle flushing with saline.

Endoprosthesis-The drainage catheter was removed and the guide wire replaced; over it was passed the permanent endoprosthesis, which was a radio-opaque 7 French Teflon catheter cut long enough to provide $5 \mathrm{~cm}$ of tubing either side of the obstruction, with multiple side holes at both ends. A "pigtail" tip may be used to reduce the possibility of dislodging it. Insertion of the prosthesis may be facilitated by making a small incision in the sphincter by means of endoscopic diathermy. The prosthesis was forced into position over the guide wire under radiological control by using a "pushing" catheter; the guide wire and pushing catheter were then removed and the position of the endoprosthesis subsequently checked by plain abdominal radiographs (fig 3 ).

\section{Case reports}

Case 1-A 68-year-old man presented with a four-week history of jaundice and weight loss. Ultrasound scanning showed dilatation of the common and intrahepatic bile ducts. Duodenoscopy showed an ulcerating carcinoma around the papilla of Vater. A catheter was passed over a guide wire through the obstruction and into the common bile duct (fig 4). The nasobiliary tube drained $700-1300 \mathrm{ml}$ of bile/day; although the serum bilirubin concentration remained constant, the alkaline phosphatase activity fell from 194 to $94 \mathrm{IU} / 1$ over 10 days, and his general condition improved. Pancreaticoduodenectomy was carried out for a tumour in the head of the pancreas; histology showed adenocarcinoma. He was well two months later.

Case 2-A 65-year-old woman presented with symptoms of obstruction of the superior vena cava secondary to a mediastinal mass. Cytological examination of pleural fluid showed undifferentiated malignant cells, and after a course of radiotherapy to the mass her symptoms resolved. Three months later she developed progressive jaundice with enlargement of the liver and a palpable gall bladder. Ultrasonography showed a dilated biliary tree, with a mass in the head of the pancreas and enlarged retroperitoneal nodes. Endoscopic retrograde cholangiography showed a stricture of the distal bile duct consistent with extrinsic compression. The pancreatic duct was normal, and brush cytology of the stricture showed normal bile-duct epithelium. Nasobiliary drainage was started and a permanent endoprosthesis inserted one week later (fig 3). Two weeks after drainage was started the serum bilirubin concentration had fallen from 165 to $52 \mu \mathrm{mol} / 1$ ( 9.6 to $3.0 \mathrm{mg} / 100 \mathrm{ml}$ ) and alkaline phosphatase activity from 205 to $89 \mathrm{IU} / 1$; ultrasonography showed progressive reduction in the calibre of the intrahepatic ducts and a patent prosthesis.

\section{Discussion}

Malignant obstruction of the bile duct is becoming more common as the incidence of pancreatic carcinoma rises. Fewer than $10 \%$ of these tumours are resectable, and operative bypass procedures may have a mortality of $25 \%$ or more. ${ }^{18}$ Intrinsic bile-duct tumours present similar problems in management. ${ }^{910}$ Hence interest is increasing in providing palliation with lessinvasive non-operative techniques. The percutaneous transhepatic approach permits preoperative external biliary drainage, which may improve operative mortality, ${ }^{311}$ and provides a route for inserting a permanent splinting endoprosthesis. ${ }^{45}$ Although complications occur with under $5 \%$ of these procedures, bleeding, pneumothorax, septicaemia, and haematobilia have all been reported. We have described an endoscopic method of providing temporary external and permanent internal drainage. The endoscopic approach is less invasive and should be safer; furthermore, removing and replacing a blocked endoprosthesis should be easier endoscopically than transhepatically. The endoscopic approach is technically more difficult, and it is not yet clear whether a guide wire can be passed through the stricture endoscopically with the same frequency as is possible using the transhepatic route.

The role of this technique in managing malignant or even benign biliary strictures has yet to be defined. Its potential for safely and rapidly relieving some of the distressing symptoms of obstructive jaundice, however, makes it worthy of further evaluation.

Requests for reprints should be sent to PBC.

\section{References}

1 Webster DJT. Carcinoma of the pancreas and periampullary region: a clinical study in a District General Hospital. Br F Surg 1975;62:130-4.

2 Buckwalter JA, Lawton RL, Tidrick RT. Bypass operations for neoplastic and biliary tract obstruction. Am f Surg 1965;109:100-6.

3 Nakayama T, Ikeda A, Okuda K. Percutaneous transhepatic drainage of the biliary tract. Gastroenterology 1978;74:554-9.

4 Burcharth F, Jensen LI, Olesen K. Endoprosthesis for internal drainage of the biliary tract. Gastroenterology 1979;77:133-7.

5 Perieras RV, Rheingold OJ, Hutson D, et al. Relief of malignant obstructive jaundice by percutaneous insertion of a permanent prosthesis in the biliary tree. Ann Intern Med 1978;89:589-93.

6 Cotton PB. Progress Report ERCP. Gut 1977;18:316-41.

7 Safrany L. Endoscopic treatment of biliary tract diseases. Lancet 1978;ii: 983-5.

${ }^{8}$ Hermann R, Cooperman AM. Current concepts in cancer. Cancer of the pancreas. New Engl F Med 1979;301:482-5.

${ }^{9}$ Bismuth H, Malt R. Current concepts in cancer. Carcinoma of the biliary tract. New Engl f Med 1979;301:704-6.

10 Lyon IM. Cholangiocarcinoma. Br 7 Hosp Med 1979;10:478-31.

11 Pollock TW, Ring ER, Oleaga JA, Freiman DB, Mullen JL, iRosato EF. Percutaneous decompression of benign and malignant biliary obstruction. Arch Surg 1979;114:148-51.

(Accepted 29 November 1979)
ONE HUNDRED YEARS AGO The question sometimes arises in court, "Is not such a wound-one, for example, penetrating the brain, or the heart, or the lungs-necessarily fatal ?" The first impulse would be to answer in the affirmative; yet a sufficient number of remarkable recoveries are on record to constitute a respectable minority of exceptions to the rule of fatality. Dr Draper, in the Boston Medical and Surgical fournal, refers to a case reported by AssistantSurgeon P F Harvey, of the United States Army, in the American fournal of the Medical Sciences for July 1879. The patient, an Indianagency physician, received a Winchester-rifle ball three inches and a quarter above and one inch behind the right external auditory meatus. The missile took a transverse direction across both hemispheres toward the left supraorbital convolution, a grooved director being easily passed in that course a distance of five inches, without, however, reaching the ball. The patient did not lose consciousness on being wounded, and complained only of "seeing stars" and of some confusion of ideas; he recovered so rapidly that, after thirty-five days of convalescence, he took a journey of ninety miles, in December, in an open buggy, alighting several times to make his way on foot through deep snow-drifts. At the end of this exertion, however, two convulsions occurred, and the wound in the head reopened. Subsequently, various threatening symptoms supervened, but they were all happily managed, and complete convalescence ensued. Six months after the wounding, the patient travelled across the plains to his home in Indianapolis, and on his arrival reported himself in excellent condition. In this connection may be mentioned an instructive paper by $\mathrm{Dr} \mathrm{H}$. R. Wharton of Philadelphia (Philadelphia Medical Times, July 9th, 1879), wherein he gives an analysis of three hundred and sixteen recorded cases in which foreign bodies were lodged in the brain. Of these 316 cases, 160 recovered and 156 died. There were 106 cases in which the intruding body was removed; the result in these instances was fatal in 34; of the 210 cases in which the foreign body was allowed to remain in the brain, 122 ended fatally in consequence, and 88 recovered. Brodie's opinion, that recovery is more apt to follow wounds of the anterior portion of the brain, is confirmed by an examination of the cases in the present series, where the foreign body penetrated the frontal bone; of these, 132 in number, 58 ended fatally and 74 resulted in recovery. The parietal bone was penetrated 58 times, with 27 deaths and 31 recoveries. The occipital bone received the lesion in 23 instances; 16 of the subjects died and 7 recovered. The foreign body entered the brain through the temporal bone 31 times; there were 12 deaths and 19 recoveries. Wounds of the orbit were by far the most fatal; there were 18 cases on record, all but one of which resulted fatally. (British Medical fournal, 1880.) 